\title{
Disseminated parvovirus infection with interstitial pneumonia and a pericardial effusion in an adult allogeneic stem cell transplant patient
}

\begin{abstract}
Parvovirus represents a common infection encountered by many people throughout child hood and adult hood. Commonly the disease will present as a simple febrile illness, rash in infants, bone marrow compromise, or even isolated arthralgias. However, in the immune compromised population, the presentation can be more a typical and can result in dissemination with major organ compromise. We present the first reported case of parvovirus pneumonia with disseminated disease in an adult allogeneic stem cell transplant patient. This case represents the importance of having a high suspicion for parvovirus infection in any transplant patient without focus on purely hematologic compromise or classic presentations. A clinician must have a high suspicion for this disease in immune compromised patients as its treatment is easily given and highly effective.
\end{abstract}

Volume I Issue I - 2015

\author{
Matthew H Richards,' Daphne Ang,' Jennifer \\ Losette, ${ }^{2}$ James Gajewski' \\ 'Oregon Health Sciences Center, Portland, Oregon \\ ${ }^{2}$ Kaiser Medical Group, Portland, Oregon
}

Correspondence: Matthew H Richards, Oregon Health \& Science University, 3।8I SW Sam Jackson Park Rd Portland, OR 97239, USA, Tel 9152049105, Email jean.klastersky@bordet.be

Received: November 15, 2014 | Published: January 13, 2015

\section{Introduction}

Parvovirus B19, after being discovered by Yvonne Cossart in the 1970 's, derived its name from the discovery that pathognomonic viral particles in human serum, after an anomalous reaction in an assay for hepatitis B, were occupying position 19 in plate $\mathrm{B} .{ }^{1}$ The virus is very common in childhood as roughly half of children at age 15 are seropositive for parvovirus B19 antibodies. ${ }^{2}$ Infection also continues to occur throughout adulthood as seropositivity approaches nearly $100 \%$ in the elderly. Clinical syndromes include erythema infectiousum, Fifth's disease, with classic erythematous cheeks and fevers in young children. In adults it is an implicated agent for a symmetric arthritis usually of the hands, wrist, knees, and ankles. The rash seen in Fifth disease and arthritis in adults in thought to be related to immune complex deposition as the main target of parvovirus particles is erythroid prescursors. ${ }^{3}$ In sickle cell patients and disease states with high red cell turnover and increased demand, aplastic crises can occur as a result of infection causing severely depressed red blood cell counts. White blood cell and platelet counts may also fall, with asplenics being the most susceptible. ${ }^{4}$ In pregnant women, placental transfer combined with an immature immune system in a fetus results in hydrops fetalis. However, most infections including those in adults are largely asymptomatic. ${ }^{5}$

Though parvovirus B19 can be detected in serum by electron microscopy or hem agglutination, the virus is most commonly detected by direct hybridization or polymerase chain reaction (PCR). The PCR technique is at least $10^{4}$ times more sensitive than hybridization and detects fewer than 10 genomes. ${ }^{6}$ Serology testing can be used to reliably diagnose infections however there is diminished specificity of some assays especially with cross-reactivity with EBV and rubella. ${ }^{7}$ As the clinical presentation can be vague, especially in transplant patients, serology must be combined with clinical suspicion or other diagnostic techniques to ensure validity. In some cases, even further confirmation with tissue usually in the form of bone marrow is needed to confirm including special staining to identify the presence of the virus.

Parvovirus B19 is a relatively rare infection in transplant patients, both solid organ and stem cell as demonstrated by the paucity of trials, prospective or retrospective. However, two retrospective trials and one case series studying the incidence of parvovirus B19 infections in solid organ transplants have been conducted..$^{8-10}$ In renal transplant patients the incidence of parvovirus viremia is $21-31 \%$ with $12 \%$ having greater than two positive serum samples. ${ }^{8,9}$ In a retrospective analysis of adult bone marrow transplant patients, an incidence of $15 \%$ having parvovirus B19 infection was measured in 60 patients receiving bone marrow transplants. ${ }^{11}$ Presentation in transplant patients can vary but the large majority present with some derivation of anemia. Nearly all case reports in bone marrow transplant patients report infection resulting in pure red cell aplasia or bone marrow failure. ${ }^{12-19}$ These manifestations happen as early as the early phase of transplantation and as late as ten years following transplant. There are case reports in solid organ transplant patients of parvovirus resulting in myocarditis ${ }^{20}$ hepatitis. ${ }^{21} \mathrm{CNS}$ vasculitis, ${ }^{22}$ and severe multiorgan failure. ${ }^{23}$ There are two reported cases of pneumonia secondary to parvovirus B19 infection, one in a heart transplant patient ${ }^{24}$ and the other in a pediatric bone marrow transplant patient. ${ }^{25}$ The spectrum of disease can vary widely but should largely be considered

\section{Case report}

The patient is a 61year old female who initially presented in May of 2008 with acute myeloid leukemia initially presenting as leukemia cutis, oral ulcers and gingival infiltration. Initial studies demonstrated a bone marrow biopsy consistent with AML (M4/M5), normal cytogenetics with no high risk features but she was considered high risk due to the extramedullary involvement with gingival infiltration confirmed on biopsy. She entered complete remission after $7+3$ induction and completed three cycles of consolidation chemotherapy. Her initial course was complicated by neutropenic fevers and thrombocytopenia with bleeding requiring recurrent platelet transfusions. This eventually resulted in alloimmunization requiring HLA-matched platelets with every transfusion. She was referred for a matched sibling allogeneic transplant in early 2009 during CR1 due to likely extramedullary disease given M5 component which has a high likelihood of extramedullary disease. 
She underwent a non-myeloablative regimen with fludarabine, busulfan and single low-dose TBI followed by infusion of 5.49 million CD34+cells per kilogram of PBSC from a matched sibling donor. Early post-transplant course was complicated by neutropenic fevers, a sinus infection on day +27 and positive blood cultures with Serratia followed by Sphingomonas paucimobilus with repeated contamination of PICC lines with this organism. Orthostatic hypotension developed and was persistent and thought to be related to calcineurin inhibitor but persisted despite switching to tacrolimus and later mycophenolate for GVHD prophylaxis. An adrenal workup showed a normal Cosyntropin stimulation test but a low basal level of cortisol. It was decided that she would be managed with fludrocortisones which was successful. Her late post-transplant course complicated by skin, gastrointestinal, and hepatic manifestations of chronic GVHD stabilized with tacrolimus and low level CMV viremia that resolved with valacyclovir and BK viruria without symptoms which persisted after a taper off immunosuppression. She had been maintained on mycophenolate $500 \mathrm{mg}$ twice daily since November of 2009 .

In late January 2010, the patient presented to clinic for follow up with a complaint of roughly one month of productive cough, progressive dyspnea, night sweats and orthostatic symptoms including a near syncope episode and had an oxygen desaturation in clinic and was found to have a mild elevation in her liver enzymes. Review of systems was otherwise unremarkable and her exam demonstrated a blood pressure of $100 / 60 \mathrm{~mm} \mathrm{Hg}$, normal oxygen saturation but dropped transiently to $85 \%$ with no objective fever and was only remarkable for a few oral ulcers, a prolonged expiratory phase, a single prominent right axillary lymph node and a scaling erythematous rash over the entire body. Parvovirus infection was suspected initially and the patient was given $35 \mathrm{~g}$ intravenous immune globulin (IVIG) in the clinic prior to admission. Initial laboratory evaluation demonstrated normal chemistries, normal blood gases, mild anemia which was stable compared to previous and recent outpatient values, normal white blood cell counts and differential, and a normal platelet count and the mild elevation in liver enzymes as mentioned. She was initially placed on meropenem for broad coverage but remained afebrile for the first three days of the hospital stay.

Pulmonary function testing was completed the following morning which showed normal lung volumes but a DLCO of $61 \%$. An echocardiogram demonstrated a moderate pleural effusion with features of tamponade. The effusion was evaluated by the cardiology team and felt it did not require drainage due to the size on imaging as well as lack of clinical signs of tamponade. Subsequently, a CT scan of the chest demonstrated bronchial thickening and nodular tree-inbud formation consistent with a viral process with diffuse but small intrathoracic lymphadenopathy and an enlarged axillary lymph node on the right. Biopsy of the axillary lymph node was undertaken in order to rule out acute B-cell lymphoproliferative syndrome. Results of this pathology demonstrated reactive lymph node changes but were negative for any features of parvovirus on microscopic examination or any other pathognomonic changes. A bone marrow biopsy was performed which demonstrated findings of parvovirus (Figure 1-4) and a serum sample was positive for PCR. Nasal washing for a common viral panel returned negative.

After her CT imaging findings returned, a bronchoscopy was completed to evaluate the etiology of those findings. Concurrently, her serum IgG levels were measured and found to be $1600 \mathrm{mg} / \mathrm{dL}$. A serum sample analyzed for galactomannan was negative along with serum CMV and EBV PCR studies. BAL washings were negative for a respiratory virus panel to include influenza, paramyxo, respiratory syncitial viruses as well as negative cultures for fungal and mycobacteria. However, the washings returned positive by PCR for parvovirus. The patient was then continued on IVIG infusions with the second infusion occurring on hospital day \#4 and \#7 for a total of 3 doses. She continued to be dyspneic, worsened by exertion and however did not ever develop signs of clinical tamponade. Also, she was initially afebrile but did have an isolated fever the day prior to the second infusion of IVIG. Repeat imaging on hospital day \#7 showed some mild bilateral pleural effusions but improvement in the parenchymal disease of the lung. Throughout her hospital course, she never developed any hematologic consequences with stable blood counts. Her liver enzyme elevation resolved after the initial day and remained within normal limits afterward.

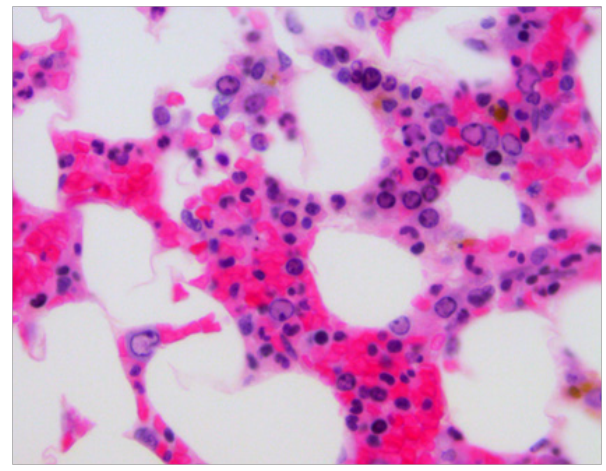

Figure I This bone marrow aspirate smear from a patient with acute parvovirus infection show markedly enlarged erythroblasts with a viral inclusion, adjacent to normal-sized hematopoietic cells

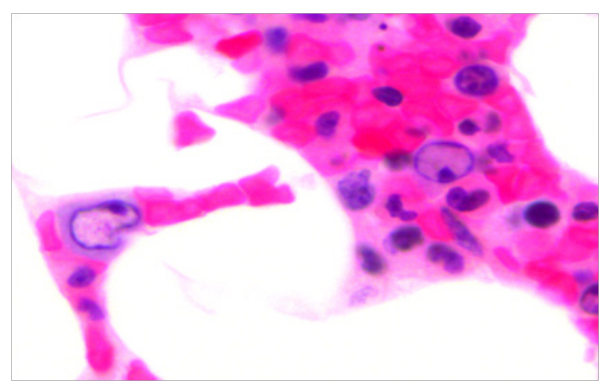

Figure 2 The bone marrow core biopsy section viewed in low power field from a patient with acute parvovirus infection shows multiple, individually dispersed, markedly enlarged erythroblasts in various stages of cytologic dissolution.

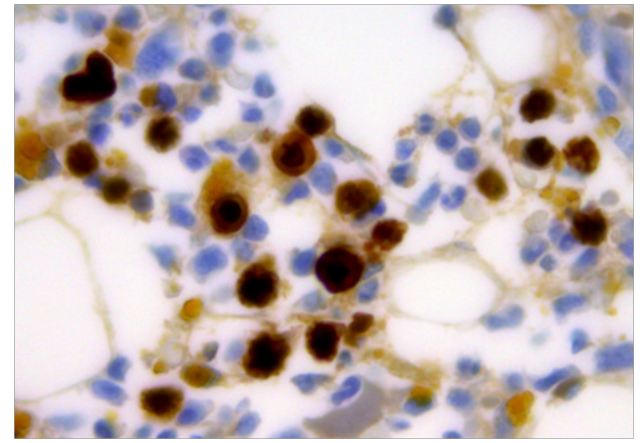

Figure 3 The bone marrow core biopsy section viewed in high power field from a patient with acute parvovirus infection shows giant erythroblasts with intranuclear inclusions. 


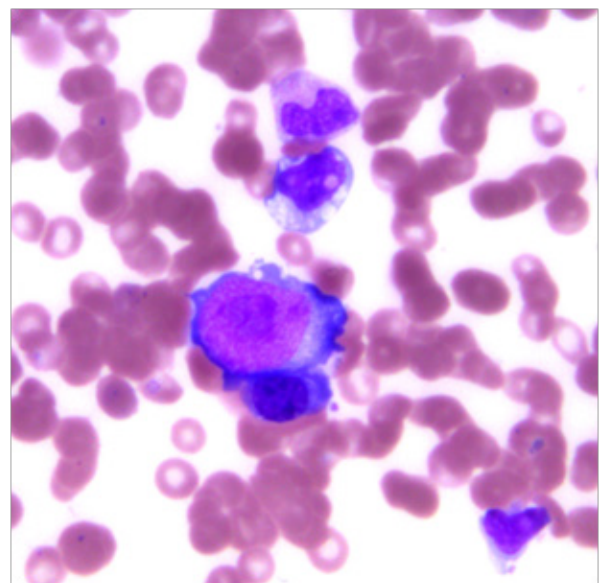

Figure 4 This bone marrow core biopsy section from a patient with acute parvovirus infection shows the virally infected erythroblasts highlighted by immunoperoxidase stain for parvovirus.

\section{Discussion}

This case represents the first reported presentation of pneumonia most likely caused by parvovirus B19 in an adult bone marrow transplant patient. Though this case is rare, it demonstrates the importance of maintaining parvovirus infection high on the differential for transplant patients. Interestingly, the patient did not have any hematologic manifestations despite the presence of the virus in the bone marrow. The variety of ways that parvovirus B19 infection can present requires clinicians to consider parvovirus for any nonspecific complaints or instances of major organ dysfunction. It should be high on the differential in any transplant patient with anemia that is new, persistent, or unexplained.

Though the main target of parvovirus B19 is erythroid precursors in the bone marrow, it may appear there is tropism within other cells from major organs including lungs, liver, and heart. There is little data to support this theory in humans but based on the case reports presented in this paper, there are multiple instances where the virus has localized and replicated within all of the major vital organs in addition to the hematopoietic tissues. Though largely a disease that is self-limited in immune competent patients, persistent or severe infection is successfully treated with prompt initiation of immune globulin in immune compromised populations. Some initial trials investigating the possibility of a vaccine were conducted; yet, none of these efforts have resulted in a successful way to enact primary prevention. However, with clinical suspicion and timely diagnosis, cases of parvovirus B19 infection in transplant patients can be easily managed and successfully treated.

\section{Acknowledgements}

None.

\section{Conflict of interest}

The author declares no conflict of interest.

\section{References}

1. Cossart YE, Field AM, Cant B, et al. Parvovirus-like particles in human sera. Lancet. 1975;1(7898):72-73.

2. Risks associated with parvovirus B19 infection. MMWR Morbidity Mortality Weekly Report. 1989;38(6):81-97.
3. Young NS, Brown KE. Mechanisms of disease parvovirus B19. N Engl J Med. 2004;350(6):586-597.

4. Saunders PWG, Reid MM, Cohen BJ. Human parvovirus induced cytopenias a report of five cases. Br J Haematol. 1986;3(2):407-410.

5. Woolf AD, Campion GV, Chishick A, et al. Clinical manifestations of human parvovirus B19 in adults. Archives of Internal Medicine. 1989;149(5):1153-1156.

6. Koch WC, Adler SP. Detection of human parvovirus B19 DNA by using the polymerase chain reaction. J Clin Microbiol. 1990;28(1):65-69.

7. Jensen IP, Vestergaard BF. Assessment of the specificity of a commercial human parvovirus B19 IgM assay. Clin Diagn Virol. 1997;7(3):133-137.

8. Cavallo R, Merlino C, Re D, et al. B19 virus infection in renal transplant recipients. J Clin Virol. 2003;26(3):361-368.

9. Ki CS, Kim IS, Kim JW, et al. Incidence and clinical significance of human parvovirus B19 infection in kidney transplant recipients. Clin Transplant. 2005;19(6):751-755.

10. Eid AJ, Brown RA, Patel R, et al. Parvovirus B19 infection after transplantation a review of 98 cases. Clin Infect Dis. 2006;43(1):40-48.

11. Schleuning M, Jager G, Holler E, et al. Human parvovirus B19-associated disease in bone marrow transplantation. Infection. 1999;27(2):114-117.

12. Itala M, Kotilainen P, Nikkari S, et al. Pure red cell aplasia caused by B19 parvovirus infection after autologous blood stem cell transplantation in a patient with chronic lymphocytic leukemia. Leukemia. 1997;11(1):171.

13. Hsu JW, Czander M, Anders V, et al. Parvovirus B19-associated pure red cell aplasia in chronic graft versus host disease. Br J Haematol. 2002;119(1):280-281.

14. Kurtzman G, Frickhofen N, Kimball J, et al. Pure red-cell aplasia of 10 years duration due to persistent parvovirus B19 infection and its cure with immunoglobulin therapy. $N$ Engl J Med. 1989;321(8):519-523.

15. Kurtzman GJ, Ozawa K, Cohen B, et al. Chronic bone marrow failure due to persistent B19 parvovirus infection. N Engl J Med. 1987;317(5):287294.

16. Cohen BJ, Beard S, Knowles WA, et al. Chronic anemia due to parvovirus B19 infection in a bone marrow transplant patient after platelet transfusion. Transfusion. 1997;37(9):947-952.

17. Arnold DM, Neame PB, Meyer RM, et al. Autologous peripheral blood progenitor cells are a potential source of parvovirus B19 infection. Transfusion. 2005;45(3):394-398.

18. Plentz A, Hahn J, Holler E, et al. Long-term parvovirus B19 viraemia associated with pure red cell aplasia after allogeneic bone marrow transplantation. J Clin Virol. 2004;31(1):16-19.

19. Hayes-Lattin B, Seipel TJ, et al. Pure red cell aplasia associated with parvovirus B19 infection occurring late after allogeneic bone marrow transplantation. Am J Hematol. 2004;75(3):142-145.

20. Jonetzko P, Graziadei I, Nachbaur K, et al. Fatal course of parvovirus B19-associated myocarditis in a female liver transplant recipient. Liver Transpl. 2005;11(4):463-466.

21. Yoto Y, Kudoh T, Haseyama K, et al. Human parvovirus B19 infection associated with acute hepatitis. Lancet. 1996;347(9005):868-869.

22. Klumpen HJ, Petersen EJ, Verdonck LF. Severe multiorgan failure after parvovirus B19 infection in an allogeneic stem cell transplant recipient. Bone Marrow Transplant. 2004;34(5):469-470.

23. Bilge I, Sadikoglu B, Emre S, et al. Central nervous system vasculitis secondary to parvovirus B19 infection in a pediatric renal transplant patient. Pediatr Nephrol. 2005;20(4):529-533. 
24. Janner D, Bork J, Baum M, et al. Severe pneumonia after heart transplantation as a result of human parvovirus B19. J Heart Lung Transplant. 1994;13(2):336-338
25. Beske F, Modrow S, Sorensen J, et al. Parvovirus B19 pneumonia in a child undergoing allogeneic hematopoietic stem cell transplantation. Bone Marrow Transplantation. 2007;40:89-91. 